\title{
A Rare Cause of Diplopia: Functional Near Reflex Spasm
}

\author{
Fatma Doğanay', Zeynep Dadacı \\ 'Department of Neurology, Mevlana University School of Medicine, Konya, Turkey \\ ${ }^{2}$ Department of Ophthalmology, Mevlana University School of Medicine, Konya, Turkey
}

\begin{abstract}
Introduction: Many serious neurological and ophthalmological diseases are reported in patients presenting to the emergency service with the complaint of diplopia. Near reflex spasm is a rare cause of diplopia, which may mimic abducens nerve palsy.

Case Report: A 27-year-old female presented to the emergency service with the complaint of diplopia and blurred vision. There was no drug usage or exposure to toxic substances. In the physical examination, convergent strabismus was observed and the pupils were miotic. Ciliary spasm was detected in the ophthalmologic examination. The results of the examinations for organic etiologies were negative. The patient recovered after the application of cycloplegic drops and the arrangement of treatment after psychiatric examinations.

Conclusion: Considering near reflex spasm in patients presenting with diplopia could save the emergency services, where time is important, from unnecessary further examinations. It should not be forgotten to pay attention to miosis because it is the most important clue evocative of this rare disease.
\end{abstract}

Keywords: Near reflex spasm, convergence spasm, diplopia

Received: 11.02.2016 Accepted: 08.04.2016 Available Online Date: 15.07.2016

\section{Introduction}

Spasm of the near reflex involves intermittent episodes of miosis, accommodation and convergence and may mimic bilateral, and occasionally, unilateral, abducens paresis (1). The triad of near reflex appears in varying degrees, rather than a fixed condition (2). The patients who have complaints of diplopia and blurred vision are esotropic, especially with respect to long-distance vision. Attention should be paid to prominent miosis. Although spasm of the near reflex may occasionally occur in patients with organic disorders $(3,4)$, it is generally psychogenic and observed in patients with conversion reactions or in anxious patients where the "spasm" is the manifestation of misdirected efforts (1).

In this report, we aimed to highlight that functional convergence spasm is one of the causes of headache, diplopia, and blurred vision.

\section{Case Report}

This case was a 27-year-old female admitted to the emergency service with the complaints of headache, diplopia, and blurred vision lasting for $1 \mathrm{~h}$. The patient expressed no previous complaints similar to this condition. Diplopia increased during longdistance vision. Personal history was negative, and there was no history of previous medical treatment. There was no drug abuse or exposure to toxic substance. In the neurological examination, pupils were miotic and isochoric and light reaction was present. There was bilateral esotropia, which was more prominent in the right eye, in primary gaze, and restricted abduction in both eyes. Other neurological and physical examinations were unremarkable. Complete blood count, liver and kidney function tests, glucose and serum electrolyte concentrations, and C-reactive protein and thyroid function tests were within normal limits. Emergency brain computed tomography and cranial magnetic resonance images showed no pathologies. There was no abnormality in the arterial and venous magnetic resonance angiographies. 

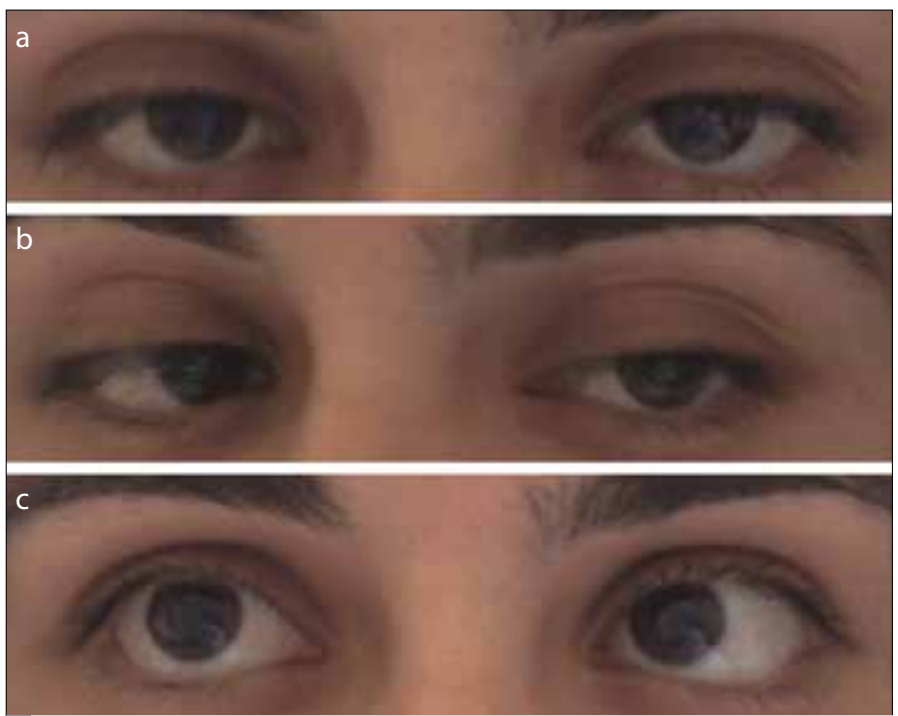

FIGURE 1. a-c. Photographs of the patient after the initiation of cycloplegia. Although the symptoms and signs are significantly subsided, both eyes are still deviated medially (a) and there is a restriction of lateral movement $(b, c)$. There is mydriasis instead of miosis because of cycloplegia.
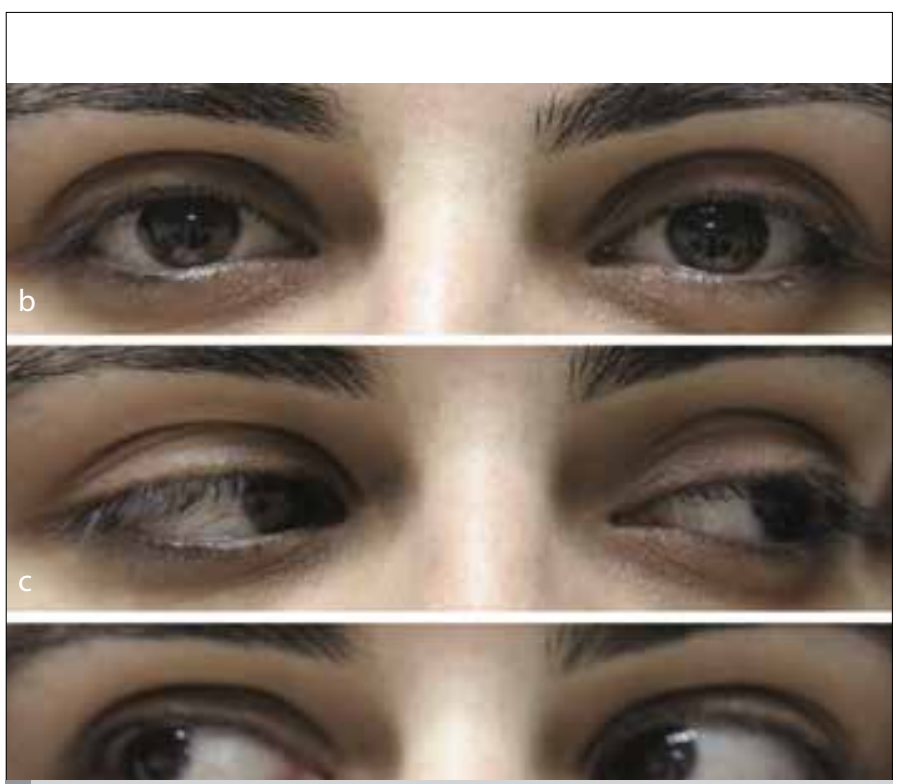

FIGURE 2. a-c. In the follow-up of the patient, it is seen that forced convergence in the primary position (a) and restriction of movement has resolved $(b, c)$.

The severity of diplopia and blurred vision fluctuated during the day.

In the ophthalmological examination, refraction of the patient without cycloplegia was -7.0 diopters (D) in both eyes and distance visual acuity corrected with $-4.0 \mathrm{D}$ in both eyes. The patient reported no previous use of glasses or contact lenses. As ciliary spasm was suspected, cycloplegia was provided with 1\% tropicamide and 1\% cyclopentolate. Cycloplegic refraction showed +0.25 D in both eyes. Anterior segment examination and fundus examination with dilata- tion was normal. The complaints of the patient decreased after the initiation of cycloplegia, but she still had restrictions in lateral gaze in the left eye (Figure 1). The patient was free of all complaints and symptoms after full cycloplegia. Her distant visual acuity after cycloplegia was 20/20 in both eyes without correction.

Anxiety signs were observed in the psychiatric examination, and treatment was administered and follow-up examinations were performed at a psychiatry clinic. There was no recurrence of the symptoms in her follow-up of 1 year (Figure 2).

\section{Discussion}

Sudden onset of diplopia and blurred vision are important symptoms suggestive of several serious neurological diseases. Myasthenia gravis; cerebrovascular accident; multiple sclerosis; ocular myopathies; $3 \mathrm{rd}$, 4th, and 6th cranial nerve palsies; some ocular diseases such as corneal pathologies and cataract; and endocrine ophthalmopathies may lead to diplopia. Possible neurological and ophthalmological diseases should be ruled out with the route of examination signs, laboratory tests, and radiological imaging. Convergence spasm, although not a common cause of diplopia, should also be considered.

Convergence spasm is rarely encountered in the neurology and ophthalmology clinics. In the literature, there are generally single case reports rather than case series. Convergence spasm is generally seen in females and young patients, as in our patient. Organic causes are rare. The association with neurological diseases and head trauma has been reported by some authors. Esotropia should be considered in the differential diagnosis. Accommodative esotropia and latent hypermetropia should be eliminated by obtaining cycloplegic refraction. Because convergence spasm generally has a course of recurrent attacks, the diagnosis may be difficult in the initial cases with a recent onset, as in our patient. Observation of miosis in the ophthalmological examination generally simplifies the diagnosis, but it can be difficult to notice. In the management process, finding the source of psychopathology should be focused and psychiatric evaluation must be demanded. Cycloplegia (cyclopentolate eye drops), negative or positive value lenses, and special covering glasses can be used to prevent accommodative spasm, and thereby, to inhibit the triad of near reflex. Recently, botulinum toxin A injection was reported to be effective in the treatment of near reflex spasm (5).

\section{Conclusion}

Considering near reflex spasm in patients presenting with diplopia could help reach a diagnosis easily and minimize the workload of the emergency department staff. Miosis should be considered because it is the most important clue of this rare disease.

Informed Consent: Written informed consent was obtained from patient who participated in this case.

Peer-review: Externally peer-reviewed.

Author contributions: Concept - F.D.; Design - F.D.; Supervision - F.D., Z.D.; Resource - F.D., Z.D.; Materials - F.D., Z.D.; Data Collection\&/or Processing - F.D.; 
Analysis\&/or Interpretation - F.D., Z.D.; Literature Search - F.D.; Writing F.D., Z.D.; Critical Reviews - F.D., Z.D.

Conflict of Interest: The authors declared no conflict of interest.

Financial Disclosure: The authors declared that this study has received no financial support.

\section{References}

1. Goldstein JH, Schneekloth BB. Spasm of the near reflex: a spectrum of anomalies. Surv Ophthalmol 1996; 40: 269-78. [CrossRef]
2. Lavin PJM, Donauhe SP. Neuro-Ophthalmology: Ocular Motor System. In Bradley WG, Daroff RB, Fenichel GM, Jankovic J, Editors. Neurology in Clinical Practice. 4nd Ed. Philadelphia: Butterworth-Heinemann (Elsevier) Press; p. 2004; 722.

3. Bohlmann BJ, France TD. Persistent accommodative spasm nine years after head trauma. J Clin Neuroophthalmol 1987; 7: 129-34.

4. Sitole S, Jay WM. Spasm of the near reflex in a patient with multiple sclerosis. Semin Ophthalmol 2007; 22: 29-31. [CrossRef]

5. Kaczmarek BB, Dawson E, Lee JP. Convergence spasm treated with botulinum toxin. Strabismus 2009; 17: 49-51. [CrossRef] 